University of Wollongong

Research Online

Faculty of Engineering - Papers (Archive)

Faculty of Engineering and Information

Sciences

$1-1-2010$

\title{
A continuously-stable linear actuation based on bending type artificial muscles
}

\author{
Rahim Mutlu \\ University of Wollongong, rm991@uowmail.edu.au \\ Gursel Alici \\ University of Wollongong, gursel@uow.edu.au
}

Follow this and additional works at: https://ro.uow.edu.au/engpapers

Part of the Engineering Commons

https://ro.uow.edu.au/engpapers/4059

\section{Recommended Citation}

Mutlu, Rahim and Alici, Gursel: A continuously-stable linear actuation based on bending type artificial muscles 2010, 732-737.

https://ro.uow.edu.au/engpapers/4059

Research Online is the open access institutional repository for the University of Wollongong. For further information contact the UOW Library: research-pubs@uow.edu.au 


\title{
A Continuously-Stable Linear Actuation Based on Bending Type Artificial Muscles
}

\author{
Rahim Mutlu ${ }^{1}$ and Gursel Alici ${ }^{1,2}$ \\ ${ }^{1}$ School of Mechanical, Materials and Mechatronic Engineering \\ ${ }^{2}$ ARC Centre of Excellence for Electromaterials Science \\ University of Wollongong, NSW, 2522, Australia
}

\begin{abstract}
Electroactive polymer actuators, also known as artificial muscles, can operate both in wet and dry media under as small as 1 Volt potential difference. This paper reports on a multi-stable linear actuation mechanism articulated with artificial muscles. Kinematic and finite element analyses of the mechanism are studied to demonstrate the feasibility of the mechanism. Experiments were conducted on a real mechanism articulated with a multiple number $(4$ or 8$)$ of electroactive polymer actuators, which had the dimensions of $12 \times 2 \times 0.17 \mathrm{~mm}$. The numerical and experimental results demonstrate that the angular displacement of the artificial muscles is accurately transformed into a rectilinear motion by the proposed mechanism. This study suggests that this multi-stable linear actuation mechanism can be used as a programmable switch and/or a pump in MEMS by adjusting the input voltage and scaling down the mechanism further.
\end{abstract}

\section{INTRODUCTION}

$\mathrm{E}$ LECTROACTIVE polymers are a class of smart materials, which can be used in numerous applications ranging from biomedical devices to micro/nano manipulation systems due to their many attractive features. One of these applications is to use them as actuators, or as famously known as artificial muscles offering the benefits of a small electrical power consumption, compliant, noiseless, small foot-print to operate, insensitive to a magnetic field, easy to fabricate, biocompatible, and a high force output to the actuator weight ratio. Further, they are very suitable to miniaturisation due to that their force to weight ratio is inversely proportional to their length. When the electrical potential is applied, an electrochemical reaction occurs and the polymer layer(s) are doped and undoped. After that, the polymer layer(s) swell and shrink converting the electrical energy into the mechanical energy. With this in mind, it is the aim of this paper to establish a non-conventional multistable linear actuation mechanism articulated with artificial muscles -electroactive polymer actuators. In this study, we exploit the smart characteristics of electroactive polymers to establish a multi-stable linear actuation mechanism. When the mechanism reaches one of its stable positions, it does not consume any electrical power. This is particularly useful if the structure is intended to remain in its any configuration for a long time, for example, as a switch.

A number of bi-stable and multi-stable mechanisms have been reported in the literature [1-3], which mainly rely on buckling of the structure. However, multi-stable configurations are achieved using multi-connected bi-stable structures, which make the mechanism more complex, and more importantly prevents its miniaturisation [4]. On the other hand, in this study, a multi-stable system can be established by using electroactive polymer actuators to create a smart structure, which adapt itself to its working conditions. The multi-stable linear actuation mechanism considered in this study does not depend on buckling theory. It can be considered as a Rapson's slide mechanism with an active hinge or a compliant mechanism. It can be used as programmable switches, valves, pumps, linear positioners and clamps etc.

\section{CONDUCTING POLYMER ACTUATORS}

Electroactive polymers derived from monomers such as pyrrole, thiophene or aniline. Their electrochemical, chemical and mechanical properties provide the fundamental basis of various devices including sensors, membranes and materials for energy storage and actuators [5-8].

A series of fabrication steps was followed to fabricate the polypyrrole (PPy) conducting polymer actuators used in this study. First, PVDF, as received, was coated with thin gold layer $\left(\sim 100{ }^{\circ} \mathrm{A}\right.$ in thickness) with sputter coating process to increase the conductivity. Second, it was immersed in a solution which contains pyrrole monomer $(0.1 \mathrm{M})$, lithium triflouromethanesulfonimide $\left(\mathrm{Li}^{+} \mathrm{TFSI}^{-}, 0.1 \mathrm{M}\right)$ and $1 \%$ water in propylene carbonate (PC). The PPy layers were galvanostatically grown from the growth solution at a current density of $0.1 \mathrm{mAcm}^{-2}$ for 12 hours on the gold coated PVDF. This process provides approximately $30 \mu \mathrm{m}$ thickness of PPy on each side of the gold coated PVDF. Therefore, the total thickness of the conducting polymer actuator was approximately $170 \mu \mathrm{m}$. [6-8]. Five layers in the polymer actuator structure are depicted in Fig. 1. This composite structure exhibits a simple bending motion like a bilayer cantilever when one-end is fixed and the other end is free to bend. 


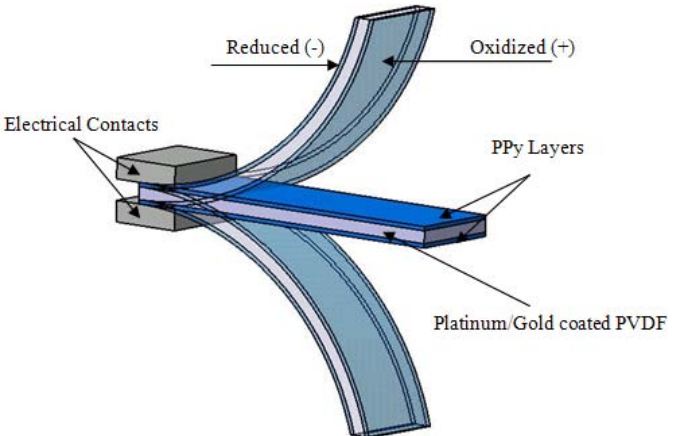

$\mathrm{PPy}^{+}\left(\mathrm{TFSI}^{-}\right)+\mathrm{Li}^{+}+\mathrm{e}^{-} \leftrightarrow \mathrm{PPy}^{\circ}\left(\mathrm{Li}^{-} \mathrm{TFSI}^{-}\right)$

(doped, oxidized state) (undoped, reduced state)

Fig. 1. Structure and operation principle of the tri-layer polypyrrole polymer actuator.

Of these layers, the gold coated PVDF in the centre is a non-conductive porous layer used for electrochemical cell separator, and also reserves electrolytic ions (e.g., Li.TFSI). Polypyrrole (PPy) layers are electroactive components [9].

The trilayer structure forms an electrochemical cell with the electrolyte stored in PVDF. When a potential difference or current is passed between the polymer (PPy) electrodes via the electrical contacts, the whole structure is charged like a capacitor: at the positively charged PPy layer is oxidised, while the negatively charged PPy is reduced. To maintain charge neutrality within the PPy layers, TFSI ${ }^{-}$anions will move from the electrolyte into the positively charged PPy layer and hence cause a volume expansion and opposite reaction occurs in the negatively charged electrode as reduction of the PPy causes it to become uncharged and a volume contraction occurs [7-9]. The overall result is that the cantilevered structure will bend towards the negative electrode/cathode, as depicted in Fig. 1.

\section{KINEMATIC ANALYSIS BASED ON EQUIVALENT LINKAGE CONCEPT}

We employ the concept of kinematically equivalent mechanisms to do the position, velocity and acceleration analyses of the multi-stable mechanism. The proposed mechanism consists of two bending type polymer actuators, acting like two active flexural joints or flexural joints with embedded actuation, allowing to slide in a common output element like a shuttle. The mechanism does not require a track to generate a rectilinear movement. The proposed mechanism and the equivalent mechanism, which is Rapson's slide mechanism, are shown in Fig. 2.

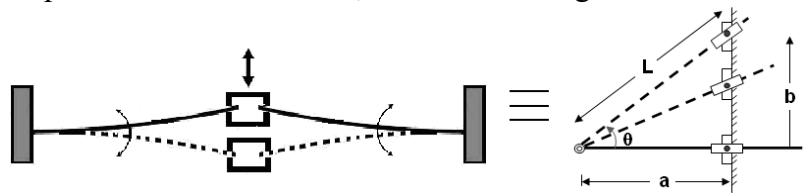

Fig. 2. Proposed linear multi-stable mechanism and its kinematically equivalent counterpart.

It must be noted that the bending behaviour of the compliant polymer actuator is represented with a revolute joint connected to a rigid link, which slides in a prismatic joint constrained to move along a straight line.

\section{A. Kinematic Model}

The equivalent mechanism illustrated in Fig. 2 is used to derive the kinematic equations and to understand the significance of the mechanism parameters and its kinematics design. This is because of the fact that it is not straightforward to model the kinematics of the real proposed mechanism as the operation principle of the conducting polymer actuators is hard to model accurately. Referring to Fig. 2, the analytical expression for the actuator can be written in terms of ' $a$ ', the provided distance, and $\theta$, the angular displacement of the rigid link;

$$
\begin{gathered}
L^{2}=a^{2}+b^{2}, \\
b=a \tan \theta \Rightarrow L=a \sqrt{1+\tan ^{2} \theta}
\end{gathered}
$$

where $\mathrm{a}$ and $\mathrm{b}$ are the horizontal and vertical coordinates of the link L while sliding along the vertical direction, respectively. From the geometry,

$$
b=L \sin \theta \Rightarrow \dot{b}=\dot{L} \sin \theta+L \dot{\theta} \cos \theta
$$

Differentiating Eq. (1) with respect to time results in

$$
\dot{b}=\dot{L} \frac{L}{b} \Rightarrow \dot{b}=\dot{L} \frac{1}{\sin \theta}
$$

Substituting Eq. (4) into Eq. (3) gives

$$
\dot{L}=L \dot{\theta} \tan \theta
$$

Substituting Eq. (5) into Eq. (4) produces

$$
\dot{b}=L \dot{\theta} \frac{1}{\cos \theta}
$$

The sliding displacement of the rigid link $\mathrm{L}$ inside the slider is

$$
\delta L=a \sqrt{1+\tan ^{2} \theta}-a=a\left(\sqrt{1+\tan ^{2} \theta}-1\right)
$$

Sliding displacement exploited in bi-stable mechanisms has been based on buckling of the compliant link to move the shuttle between two positions upon the application of a force on the compliant element. In contrary, in this study, we allow the compliant link to slide in the shuttle and create a multi-stable mechanism, depending on the input voltage which is on the order of $1-1.5 \mathrm{~V}$ maximum. The smaller is the input voltage, the shorter is the linear movement of the shuttle.

\section{B. Kinematic Analysis based on Working Model of Mechanism}

The working model of the multi-stable mechanism consists of a short cylindrical support frame to which two cantilever beams are connected symmetrically. Another pair of cantilever beams is also attached to improve its positioning stability and increase the linear force output. These four cantilever beams, corresponding to four bendingtype polymer actuators, are connected to a short cylindrical shuttle to complete the mechanism. 
For the cantilever beams with the dimensions of $12 \times 2 \times 0.17 \mathrm{~mm}$ and input bending displacement of 4.392 degrees, the rectilinear displacement of the inner cylinder of the shuttle is calculated for 5 seconds. The other parameters considered in the kinematic analysis are listed in Table 1.

TABLE 1

PARAMETERS USED IN THE KINEMATIC ANALYSIS OF THE WORKING MODEL

\begin{tabular}{ll}
\hline Input Voltage & $2 \mathrm{~V}$ (peak to peak) \\
Frequency & $0.1 \mathrm{~Hz}$ \\
Angle of actuator & $2 \times(2.196)=4.392$ degrees \\
Time & 5 second \\
Cantilever Dimension & $12 \times 2 \times 0.17 \mathrm{~mm}$ \\
\hline
\end{tabular}

Sequence of the configuration of the mechanism during the 5 second movement is illustrated in Fig. 3. The bending angle of the polymer actuator varies from $0^{\circ}$ to $4.392^{\circ}$. As seen in Fig. 4, the polymer actuator makes $-2.196^{\circ}$ with the horizontal axis at the initial state and $2.196^{\circ}$ at the last state. Therefore, it bends $4.392^{\circ}$ in total. This angular displacement was measured from a cantilevered conducting polymer actuator with the same geometric dimensions under a $1 \mathrm{~V}$ step input.
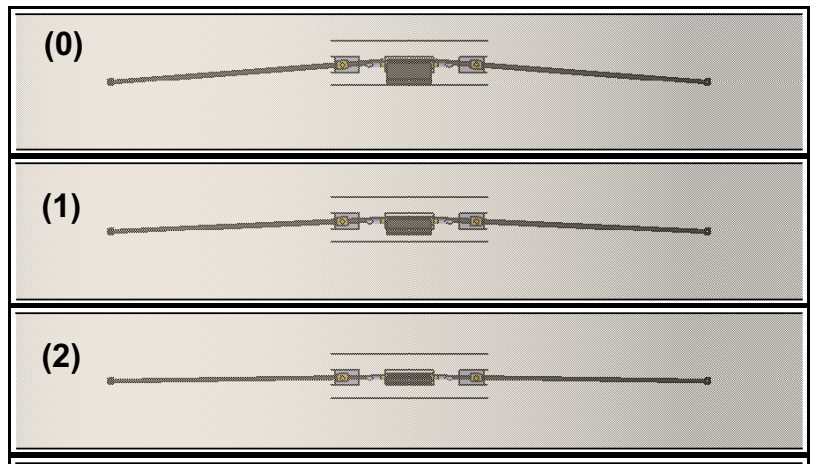

(3)

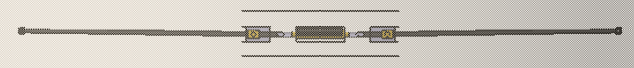

(4)

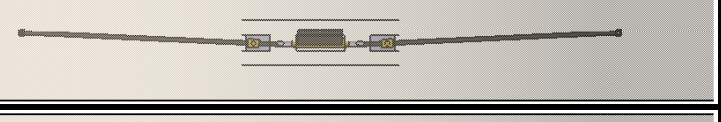

(5)

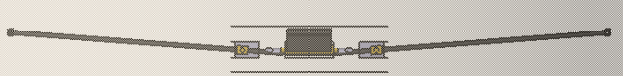

Fig. 3. Steps of the mechanism (from top; $0 \mathrm{~s}$ to $5 \mathrm{~s}$ with 1 second intervals).

The variation of the bending angle, sliding displacement of the polymer actuator inside the shuttle (Eq.7), which is the change in the length of the polymer actuator, and the vertical displacement of the middle cylinder with time are presented in Fig. 4.

Also, the rectilinear displacement of the shuttle, change in the actuator length, and their derivates are calculated from
Eqs. 1-7 and are plotted in Fig. 4. These results demonstrate that the design concept and the kinematic modelling approach are validated.
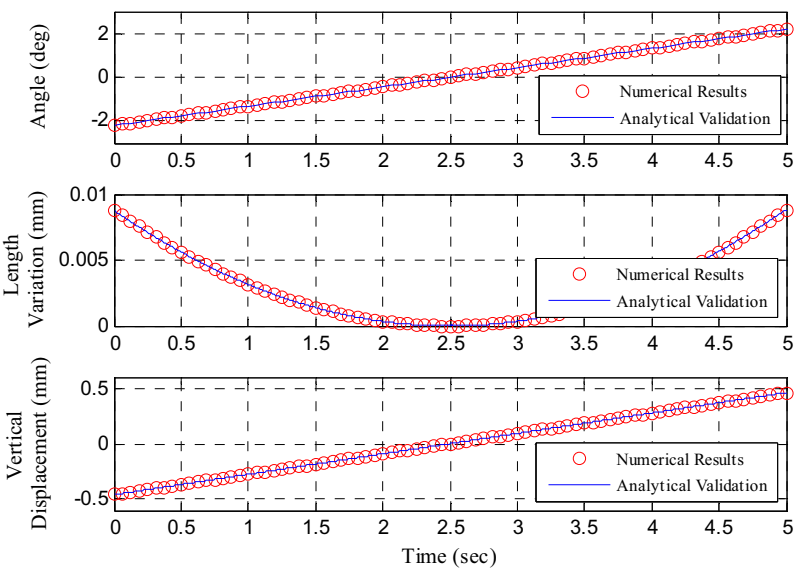

Fig. 4. Bending angle of the polymer actuator (top), change in the length of the polymer actuator (middle), and rectilinear displacement of the middle cylinder versus time (bottom).

\section{FINITE ELEMENT ANALYSIS OF MULTISTABLE MECHANISM}

Finite element analysis is used to characterize the rectilinear displacement and force output under static loading. The dimensions of the cantilevered polymer actuators, and Young's Moduli of PPy and PVDF are given in Table 2 [12].

TABLE 2

SPECIFICATIONS FOR THE PPY AND PVDF LAYERS

\begin{tabular}{lcccc}
\hline \multicolumn{4}{c}{ Dimensions } & Elasticity \\
& Length(mm) & width $(\mathrm{mm})$ & thickness $(\mu \mathrm{m})$ & $\begin{array}{l}\text { Modulus } \\
(\mathrm{MPa})\end{array}$ \\
\hline PPy & 12 & 2 & 110 & 200 \\
\hline PVDF & 12 & 2 & 30 & 117 \\
\hline \hline
\end{tabular}

A. Quasi-Static Analysis of the Finite Element Model with Four Polymer Actuators.

As the operation principle of conducting polymer actuators is based on the movement of ions in and out of the active polymer layers, the actuation gradient along the thickness direction is assumed to be constant along the actuator length. This causes an internally induced bending force generating the tip displacement. This actuation effect can be represented by a uniformly distributed load or a pressure distribution on the surface of the actuator [13]. We have modelled the bending behaviour of cantilevered-type composite polymer beams with the same dimensions and the modulus of elasticity such that it produces the same deflection as the experimental conducting polymer actuators. A uniformly distributed load is applied onto the wide surface of the polymer actuators, as illustrated in Fig. 5. A set of simulation results corresponding to a PPy polymer actuator under a range of step input voltages $(0.1-1.0 \mathrm{~V})$ is obtained. 


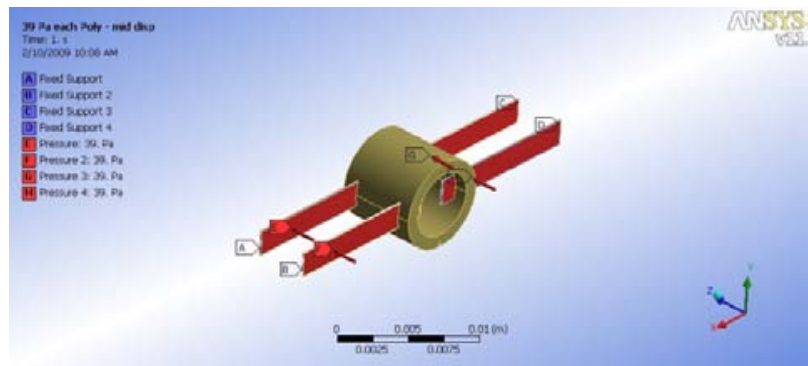

Fig. 5. Constraints and conditions of the multi-stable linear actuation mechanism's model with four polymers.

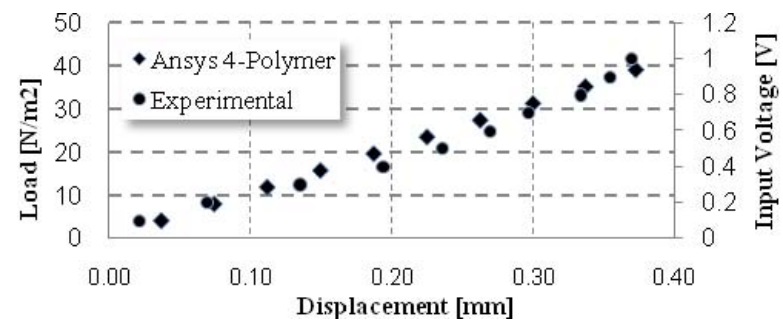

Fig. 6. PVC middle cylinder's displacement of the finite element model with four polymer actuators.

It is found that a distributed load of $39 \mathrm{~N} / \mathrm{m}^{2}$ on the simulated cantilevered-beam can generate similar displacement (tip deflection) as the experimental conducting polymer actuator under $1.0 \mathrm{~V}$, as shown in Fig. 6 . Therefore, a pressure of $39 \mathrm{~N} / \mathrm{m}^{2}$ is used to estimate the bending displacement of the tri-layer PPy polymer actuators in the mechanism.

Also, the force output of the mechanism with four polymer actuators is estimated and is presented in Fig. 7 for comparison with the experimental force output. These results show that the finite element modelling approach is accurate enough to predict the real output force.

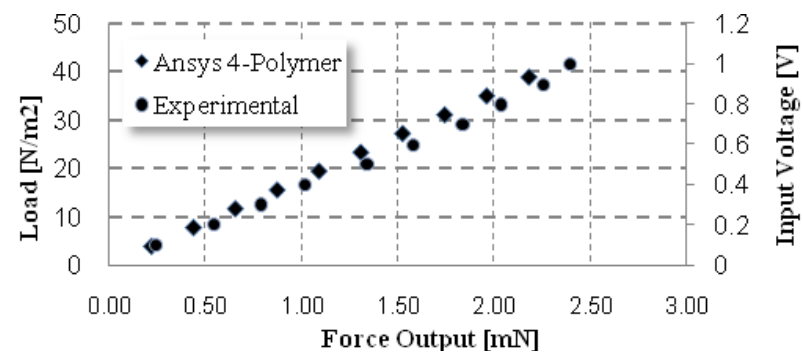

Fig. 7. Simulated and experimental force outputs of the mechanism with four polymer actuators measured from the front face of the PVC middle cylinder.

B. Quasi-Static Analysis of the Finite Element Model with Eight Polymer Actuators.

The same numerical data are used to generate simulation results for the mechanism with eight polymer actuators. 39 $\mathrm{N} / \mathrm{m}^{2}$ is applied onto each polymer actuators which are fixed from one end and are allowed to slide in the PVC middle cylinder while the actuators bend, as illustrated in Fig. 8.

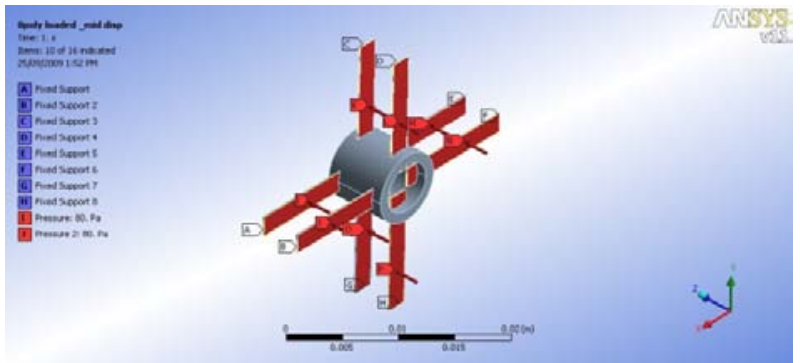

Fig. 8. Constraints and conditions of the multi-stable linear actuation mechanism with eight polymers.

Even though the same amount of displacement is generated by both mechanisms, the blocking force generated by the mechanism with eight polymer actuators is two times greater than the force generated by the mechanism with four polymer actuators, as illustrated in Fig. 9. The rectilinear displacement of the mechanism under eight polymer actuators is depicted in Fig. 10.

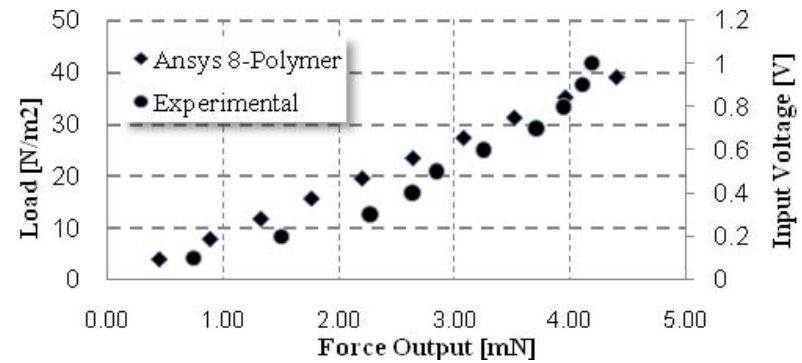

Fig. 9. Simulated and experimental force outputs of the mechanism with eight polymer actuators measured from the front face of the PVC middle cylinder.

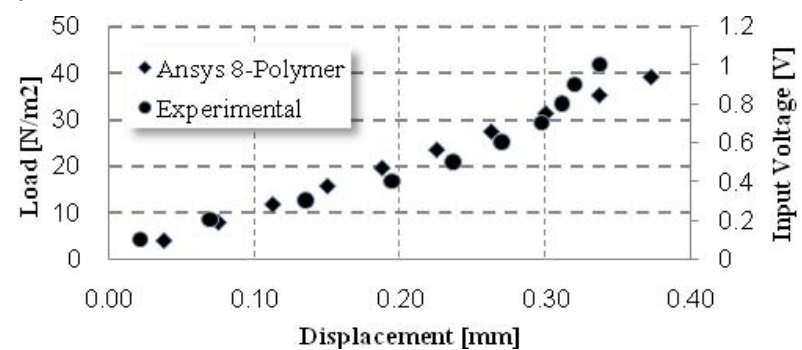

Fig. 10. Rectilinear simulated and experimental displacements of the mechanism with eight polymer actuators

\section{EXPERIMENTAL RESULTS AND DISCUSSION}

Experiments were conducted to evaluate the rectilinear displacement and force outputs for two cases; the multistable linear actuation mechanism with (i) four and (ii) eight conducting polymer actuators. The displacement and force output experiments were conducted for two cases; (i) $2.0 \mathrm{~V}$ peak to peak input voltage with a variable frequency range of 0.1 to $1.0 \mathrm{~Hz}$, and (ii) at a constant frequency of $0.1 \mathrm{~Hz}$ with a variable input voltage range of $0.1 \mathrm{~V}$ to $1.0 \mathrm{~V}$.

The polypyrrole polymer actuators have the dimensions of $12 \times 2 \times 0.17 \mathrm{~mm}$; the same dimensions of the actuators used in the finite element analysis and kinematic analysis. 


\section{A. Experimental Setup}

The experimental setup consists of the tri-layer conducting polymer actuators, and a cylindrical frame and a cylinder manufactured from PVC assembled in the centre of the cylindrical frame with 4 or 8 cantilevered conducting polymer actuators. The polymer actuators were fabricated using the procedure summarised in Section 2 and then cut into the dimensions $12 \times 2 \mathrm{~mm}$ which are length and width of the polymer actuators, respectively. Permanent magnets were used to be able to clamp the polymer actuators and as electrodes to apply input voltage, as illustrated in Fig. 11.

To actuate the conducting polymer actuators and to obtain displacement and force output data, eDAQ Chart software installed on a computer (PC) with a DAQ was used to acquire and control the data flow in the experiments. Illustrative data acquired is presented in Fig. 12.

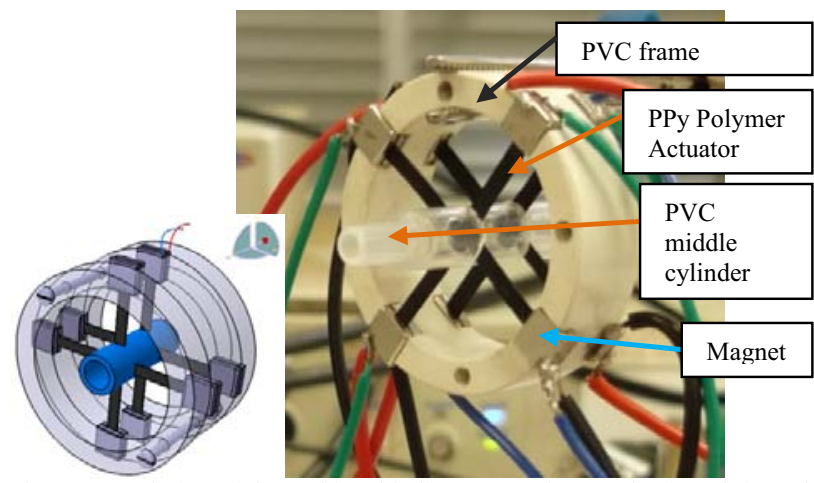

Fig. 11. 3D design of the multi-stable linear actuation mechanism (left) and manufactured multi-stable linear actuation mechanism (right).

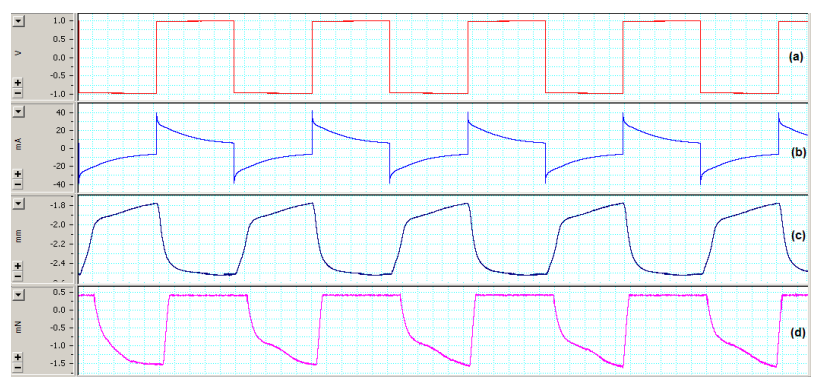

Fig. 12. Typical displacement and force outputs of the multi-stable linea actuation mechanism with 4 polymer actuators under a $1 \mathrm{~V}$ input voltage $(2 \mathrm{~V}$ peak to peak). The input voltage, the current passed, the displacement of the PVC middle cylinder and the blocking force are shown in plots (a), (b), (c), (d), respectively.

Also, a function generator was used to apply variable frequency and variable input voltages. Schematic representation of the key components is shown in Fig. 13. Two measurement systems were used which are displacement measurement and force measurement systems. The displacement measurement system is surrounded by a thicker dashed line and the force measurement system is surrounded by a thinner dashed line, as depicted in Fig. 13. Also, Displacement and force measurement system are shown in Fig. 14.

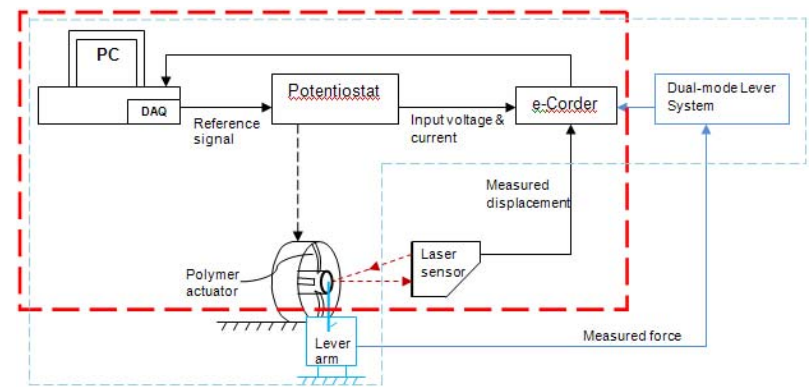

Fig. 13. Schematic representation of the experimental system.

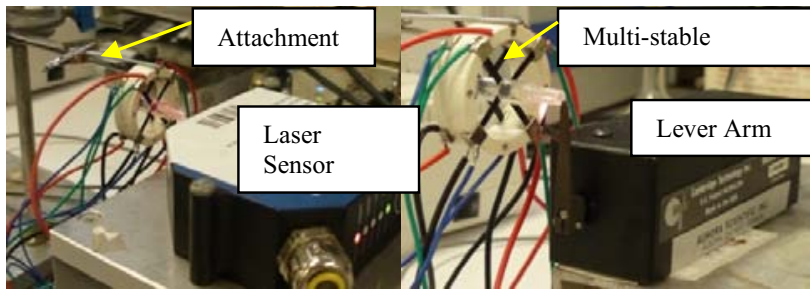

Fig. 14. Displacement measurement (top), force output measurement (bottom) of the multi-stable linear actuation mechanism with eight polymer actuators.

Experiments were conducted to observe rectilinear displacement of the multi-stable linear actuation mechanism which was obtained between two maximum bending positions of the polymer actuators measured from the PVC middle cylinder of the mechanism by a laser displacement sensor. Also, the force output results were obtained, which are measured from the PVC middle cylinder by a dual-mode lever arm system. Displacement and force output results are given in Figs. 15-16 and Figs. 17-18, respectively.

With reference to Figs. 15 and 17, the displacement and the output force decrease linearly (approximately) with the input frequency. This can be explained by the fact that the movement of dopant ions and solvent molecules requires certain time to move in and out of the PPy layers during the redox process. Obviously, with high input frequencies, there is not sufficient time for the ions and the molecules to reach deep into the polymer layer to generate more force [14]. Also, the experiments with 4 and 8 polymer actuators show $\sim 18 \%$ difference in the displacement output under a range of frequency $(0.1-1.0 \mathrm{~Hz})$ as shown in Fig. 15. However, the trend of the data measured is the same which suggests that this may be due to systematic errors. In addition, the friction between polymer surface and the cavities, in which polymer actuators are inserted and slide during operation, might cause a variation in different measurement times due to ragged surface of the actuators and the cavities.

In regard to the numerical and experimental results depicted in Sections 3,4 and 5, the multi-stable linear actuation mechanism articulated with four and eight polypyrrole polymer actuators transforms the actuators' angular movement into a rectilinear movement which is generated by the PVC middle cylinder. Also, the numerical kinematic and finite element analyses results and corresponding experimental results are in good agreement to 
suggest that the mechanism design concept is suitable to generate a rectilinear motion.

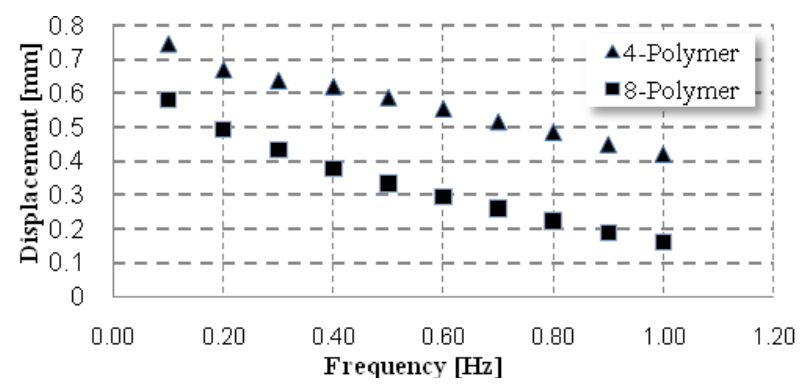

Fig. 15. Displacement results of the experiments measured from the PVC middle cylinder in a range of frequency $(0.1-1.0 \mathrm{~Hz})$ under $1.0 \mathrm{~V}$ input voltage.

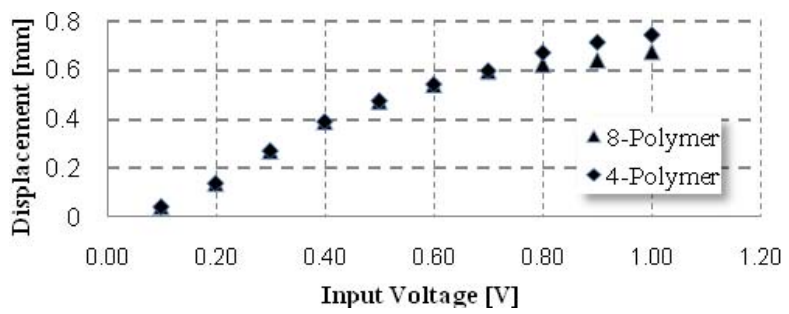

Fig. 16. Displacement results of the experiments measured from the PVC middle cylinder under a range of input voltage $(0.1-1.0 \mathrm{~V})$ in $0.1 \mathrm{~Hz}$ frequency.

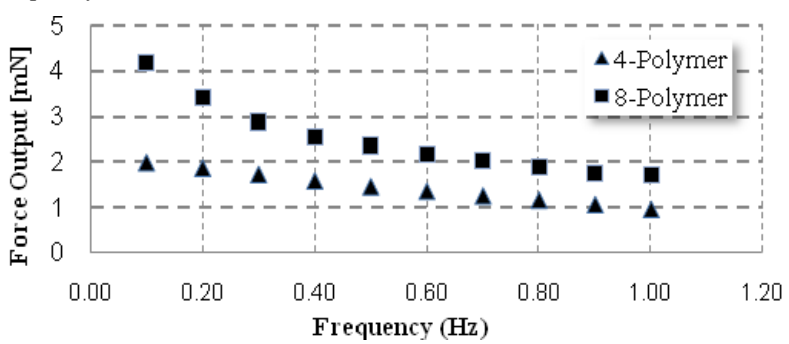

Fig. 17. Force output results of the experiments measured from the PVC middle cylinder under $1.0 \mathrm{~V}$ input voltage and a range of frequency $(0.1-$ $1.0 \mathrm{~Hz})$.

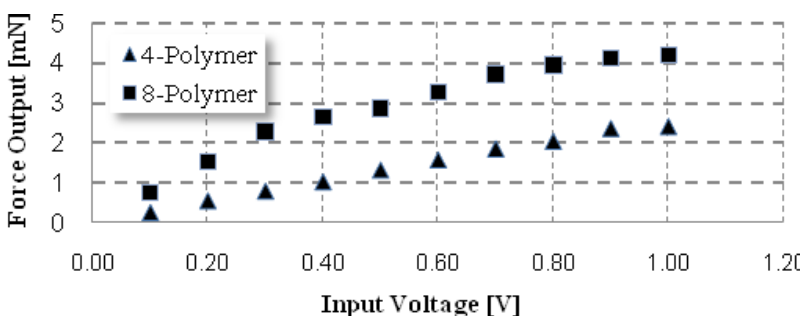

Fig. 18. Force output results of the experiment measured from the PVC middle cylinder under $1.0 \mathrm{~Hz}$ frequency and a range of input voltage (0.1 $1.0 \mathrm{~V})$.

\section{CONCLUSIONS AND FUTURE WORK}

To the best our knowledge, this is the first time that trilayer polypyrrole conducting polymer actuators were tested in such a mechanism, which transforms the bending or angular displacement of the conducting polymer actuators, into a rectilinear displacement. The performance characteristics of the multi-stable linear actuation mechanism were examined. The numerical and corresponding experimental results are in good agreement. The results show that the higher is input voltage, the larger rectilinear stroke of the mechanism.

Future work includes miniaturising the multi-stable linear actuation mechanism based on micro-sized polymer actuators [8]. The displacement and force outputs can be adjusted to meet the application requirement. For example, it can be used as a micro-pump in MEMS by adjusting the input voltage and its frequency.

\section{ACKNOWLEDGEMENTS}

This work was supported in part by the ARC Centre of Excellence for Electromaterials Science (CE0561616), and Centre for Intelligent Mechatronics Research.

\section{REFERENCES}

[1] Wang D, Pham H \& Hsieh Y 2009, 'Dynamical switching of an electromagnetically driven compliant bistable mechanism', Sensors and Actuators, vol.149, pp.143-51.

[2] Sonmez U \& Tutum CC 2008, 'A Compliant Bistable Mechanism Design Incorporating Elastica Buckling Beam Theory and PseudoRigid-Body Model', ASME Journal of Mechanical Design, vol.130, pp.042304-14.

[3] Receveur RAM, Marxer CR, Woering R, Larik VCMH \& Rooij NF 2005, ' ', IEEE/ASME Journal of Microelectromechanical Systems, vol.14, no.5, pp.1089-98.

[4] Santer M \& Pellegrino S 2008, 'Compliant multistable structural elements', International Journal of Solids and Structures, vol.45, pp.6190-204.

[5] Alici G, Spinks G, Huynh NN, Sarmadi L \& Minato R 2007, 'Establishment of a biomimetic device based on tri-layer polymer actuators - propulsion fins: Establishment of a biomimetic device', $J$. Bioinspiration \& Biomimetics, 2 S18-30.

[6] Alici G \& Shirinzadeh B 2003, 'Kinematics and stiffness analyses of a flexure-jointed planar micromanipulation system for a decoupled compliant motion', Int. Conference on Intelligent Robots and Systems, vol.4, pp.3282-87

[7] Wu Y, Alici G, Spinks GM \& Wallace GG 2006, 'Fast tri-layer polypyrrole bending actuators for high speed applications', Synthetic Metals, vol.156, no.16-17, pp1017-22.

[8] Alici G, Devaud V, Renaud P \& Spinks GM 2009, 'Conducting polymer microactuators operating in air', J. Micromechanics and Microengineering, vol.19, 025017.

[9] Mutlu R \& Alici G, 'Artificial Muscles with Adjustable Stiffness', Journal of Smart Materials and Structures, October 2009. (Under review)

[10] Alici G \& Huynh NN 2006, 'Predicting force output of trilaye polymer actuators', Sensors and Actuators, vol.132, no.2, pp.616-25.

[11] Tsai LW 2000, Mechanism design: Enumeration of kinematic structures according to function, CRC Press, Florida.

[12] John SW 2008, 'Modeling and Control of Polymer Actuators', PhD thesis, School of Mechanical, Materials and Mechatronic Engineering, University of Wollongong.

[13] Alici G, 'An Effective Modeling Approach to Estimate Nonlinear Bending Behaviour of Cantilever Type Conducting Polymer Actuators', Sensors and Actuators: B. Chemical, vol.141, no.1, pp.284-92.

[14] Alici G \& Huynh NN 2007, 'Performance Quantification of Conducting Polymer Actuators for Real Applications: a Microgripping System', IEEE/ASME Transactions on Mechatronics, vol.12, no. 1 , pp.73-84. 